\title{
Letter
}

\section{Mode decomposition of multimode optical fiber beams by phase-only spatial light modulator}

\author{
M D Gervaziev ${ }^{1,2}$, I Zhdanov ${ }^{1,2}$, D S Kharenko ${ }^{1,2}$, V A Gonta ${ }^{1,2}$, V M Volosi ${ }^{1,2}$, \\ E V Podivilov ${ }^{1,2}$, S A Babin ${ }^{1,2}$ and S Wabnitz ${ }^{1,3}$
}

\author{
${ }^{1}$ Novosibirsk State University, Novosibirsk 630090, Russian \\ ${ }^{2}$ Institute of Automation and Electrometry SB RAS, Novosibirsk, Russian \\ ${ }^{3}$ DIET, Sapienza University of Rome, Via Eudossiana 18, 00184 Rome, Italy
}

E-mail: kharenko@iae.nsk.su

Received 7 November 2020

Accepted for publication 25 November 2020

Published 18 December 2020

\begin{abstract}
Multimode optical fibers (MMF) recently attracted a renewed attention, because of their potential for spatial division multiplexing, medical imaging and high-power fiber lasers, thanks to the discovery of new nonlinear optical effects, such as Kerr beam self-cleaning, spatiotemporal mode-locking, and geometric parametric instability, to name a few. The main feature of these effects is that many transverse modes are involved in nonlinear interactions. To advance our understanding, it is necessary to analyse the modal content of beams at the output of MMFs. In this work, based on a computer digital holography method using a phase-only spatial light modulator (SLM) as a correlation filter, we experimentally demonstrate a method of mode decomposition involving a large $(\simeq 80)$ number of fiber modes. To obtain this, we carried out a SLM calibration, and numerically investigated the most critical parameters which affect the fidelity of the decomposition, by comparing experimental and reconstructed beam patterns in both the linear (speckled structures) and in the nonlinear (self-cleaned beams) propagation regime.
\end{abstract}

Keywords: beam characterization, spatial light modulation, mode decomposition, GRIN multi-mode fibers

(Some figures may appear in colour only in the online journal)

\section{Introduction}

Research on single mode optical fibers (SMFs) for communications and fiber laser applications has been progressing for a long time. Unlike multimode fibers (MMFs), SMFs have the advantage of preserving the high quality of laser beams at their output [1]. As a matter of fact, the quality of a beam at the fiber output is inversely proportional to the number of fiber modes [2]. Nevertheless, interest in MMFs has been renewed by the recent discovery of novel and intriguing nonlinear effects in GRIN fibers. For example, Kerr beam self-cleaning [3], and supercontinuum generation with high pulse powers and spectral brightness [4, 5], permit to recover the input laser beam quality at the fiber output, in spite of the large number of propagating fiber modes.

In order to characterize the quality of optical beams, the standard method involves the measurement of their $\mathrm{M}^{2}$ parameter. This parameter measures the divergence of the beam, relative to that of a diffraction-limited, or Gaussian beam. However, for multimode beams it is necessary to evaluate the distribution of their energy among the individual linear modes of the guiding structure. This calls for methods to perform a mode decomposition (MD), which provide a more effective approach to qualitatively and quantitatively characterize 
the beam quality, rather than the $\mathrm{M}^{2}$ parameter. Existing MD methods are based on genetic algorithms [6], adaptive optics [7], or digital holograms produced by spatial light modulators (SLMs) [8]. In addition, there are groups of decomposition methods based on the spatiotemporal (TERMITES) [9] or spatial and spectral (so called $S^{2}$ ) [10] distributions of the output beams. Each of these methods is quite complex, and it has its own range of applicability. Specifically, the use of the $S^{2}$ technique has been proposed as an effective way to characterize beams carried by large-mode-area fibers: the method permits to quantify the number, type, and relative power level of higher-order-modes supported by these fibers. The $S^{2}$ method works in the linear propagation regime, and it does not take into consideration the presence of nonlinear interactions between the fiber modes. Whereas the TERMITES method involves the measurement of the 3D field by means of self-reference measurements: it requires an iterative algorithm, including the task of reverse decomposition. Nonetheless it can be successfully applied for mode-resolved measurements [11].

In our approach, we show that an SLM can lead to the direct non-iterative MD of radiation at the output of a GRIN MMF, in terms of its individual linear modes. In addition, our MD method also permits to reveal the presence of nonlinear modal interactions. SLMs have many applications: for instance, in a review article [12], the authors listed the different of possibilities of using SLMs: (i) for creating phase masks in order to obtain different lasers modes, or fields with predefined configurations; (ii) for measuring the $\mathrm{M}^{2}$ beam quality parameter; (iii) for performing the MD of laser beams. However, so far all works related to MD were restricted to considering beams propagating in few-mode MMFs only (i.e. with a maximum ten modes in total). Therefore, the applicability of MD techniques to analyze beams comprising a relatively large (up to several tens) number of modes remains still questionable.

In this Letter, we introduce, numerically validate, and successfully experimentally demonstrate a MD method based on digital holography. Our aim is to realize a setup for the MD of beams propagating in GRIN MMFs, including up to one hundred of modes. We carry out a theoretical study for describing phase masks calculations, which permit to reconstruct both mode amplitudes and intermodal phases. We also investigate in details the influence of parameters such as the SLM resolution (expressed as the pixel size of the fundamental GRIN fiber mode), and the accuracy of the position of the center of the first diffraction maximum. By our experimental setup, we demonstrate the MD of low-power speckled beams at the output of a GRIN MMF. The MD method also permits to reveal the change of output mode distribution as a result of nonlinear mode interactions, which occur when the input beam power grows larger. The validity and accuracy of the method was confirmed, by comparing initial and reconstructed intensity profiles.

\section{SLM calibration}

Before carrying out the MD experiments, it is necessary to properly calibrate the SLM, in order to achieve the required
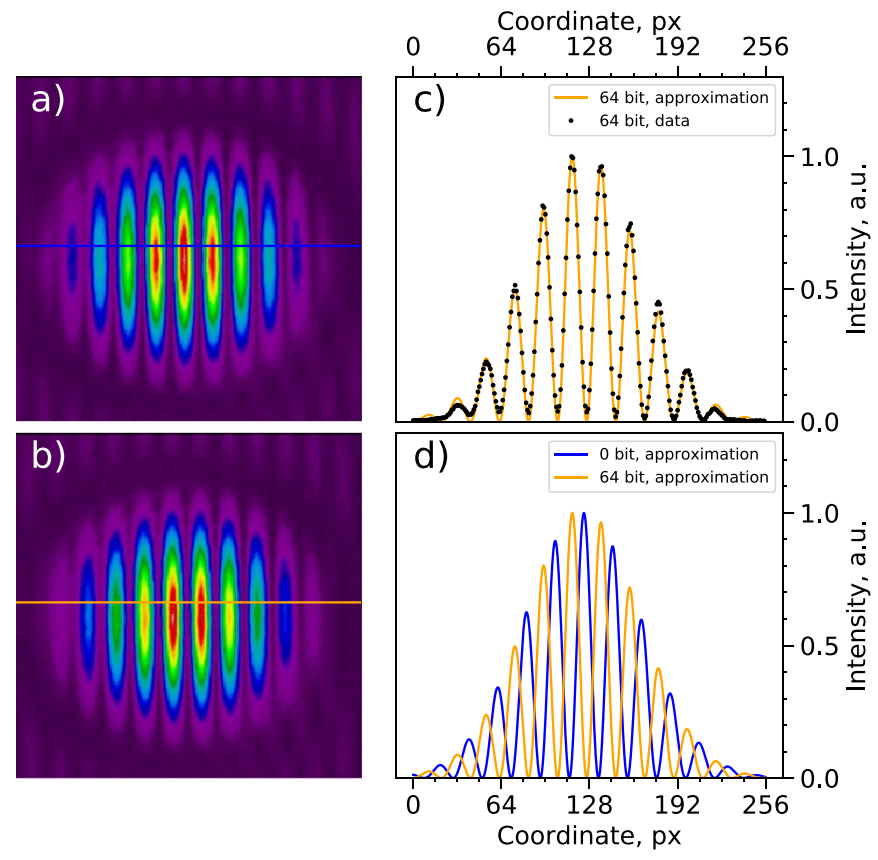

Figure 1. Two examples of observed images of interfering fringes (a), (b) for different set values (0 and 64), showing the approximation of the experimental image section (c), and the comparison of approximated sections (d).

phase-shift between pixels. In our work, we used a HOLOEYE Pluto-2 reflective-phase-only liquid crystal on silicon SLM, with a $1920 \times 1080$ resolution display, and a pixel size of $8 \mu \mathrm{m}$, which allows for generating 256 levels phase masks. All of the reported results have been obtained with this SLM. Calibration experiments are aimed at determining the phase response curve, and the values of the so-called dark and bright voltages $\left(V_{D}\right.$ and $V_{B}$, respectively), for generating a linear phase shift over a suitable range of control voltages. Thus, the input signal into an SLM is a digital signal, containing an 8 bit signal for each pixel, which is converted in a voltage between $V_{D}$ and $V_{B}$. For determining the phase, it was necessary to conduct an interferometric experiment, which permits to measure the relative phase shift between different parts of the screen. A standard diffraction experimental scheme with two hole apertures, as described in [13], was chosen for this purpose. The main idea of this experiment is to interfere two parts of the initial beam with different phase shifts: these parts are produced by two sections of the SLM display. For the spatial division of two incident rays, we used the aperture provided by two circular hollows in on opaque screen. Fringes that result from the interference of these two light sources can be observed on the camera (see figure 1). The position of fringe maxima depends on the phase difference between the two sections of the SLM display (figures 1(a) and (b)). In this way, by setting some constant value for one section, while varying the other voltage from $V_{D}$ to $V_{B}$, the phase response curve can be measured with high accuracy. For each step, pictures were analyzed for extracting the structure period $v$ and the relative phase $\phi$, by fitting the image cross-section near its center with the function 


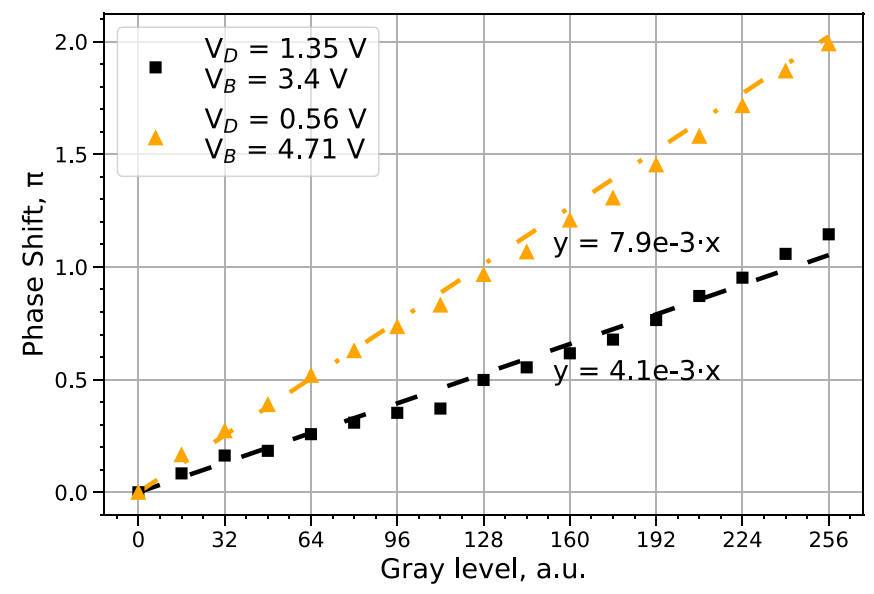

Figure 2. SLM phase shift for the $2 \pi$ configuration $\left(V_{D}=0.56 \mathrm{~V}\right.$ and $\left.V_{B}=4.71 \mathrm{~V}\right)$ and $\simeq 1.16 \pi\left(V_{D}=1.35 \mathrm{~V}\right.$ and $\left.V_{B}=3.40 \mathrm{~V}\right)$, approximated with linear functions.

$$
f_{\text {fit }}(x)=I_{0} \mathrm{e}^{-\frac{\left(x-x_{0}\right)^{2}}{w^{2}}} \sin ^{2}(v x-\phi)
$$

where $\phi$ and $v$ are the fitting parameters to be extracted (see figure 1(c)). On figure 1(d) one can easily appreciate the shift of interference fringes for different sets of parameter values: this shift corresponds to the phase shift in radians produced by the display. Changing bright and dark voltages led to determining the phase shift range. It is important to note that the $\left(V_{D}\right.$ and $V_{B}$ ) voltages are discrete and interdependent parameters, which can only be varied across a limited set of values (for our SLM device).

Finally, two calibration curves have been found for a total phase shift of up to $2 \pi$ (with a deviation of the maximum $\sim 0.5 \%$ ) ) or $\geqslant \pi$ (see yellow and black curves in figure 2 , respectively). The curve corresponding to a $2 \pi$ phase shift is well approximated by a straight line: the discrepancy between experimental data and the linear fit approximation could be expressed as follows

$$
S=\sum_{i}\left(y_{i}-f\left(x_{i}\right)\right)^{2}
$$

where $f\left(x_{i}\right)$ is the fitting function.

Another option to control the output phase is adjusting the curvature of the phase shift, which depends on digitally set values. This can be done via the so-called gamma curve, that is used to compensate non-linear response of liquid crystals to an applied voltage. In all experiments, the standard factory gamma curve obtained for the $1064 \mathrm{~nm}$ wavelength was used. Increasing its precision for fitting and control will provide another option for further improvements of the accuracy of the MD. Linear fitting yields $S_{\text {lin }}=0.033$ for the $2 \pi$ shift. As shown below, phase shifts ranging from 0 to $1.16 \pi$ are sufficient for our experiments. Therefore, we re-calibrated our SLM by using $V_{D}=1.35 V$ and $V_{B}=3.4 V$ (see the black curve in figure 2). The discrepancy with respect to a straight line fit was $S_{\text {lin }}=0.036$. These parameters were used in all of the subsequent experiments.

\section{Theoretical foundation}

In [8], the authors demonstrated an MD method, based on the use of a phase-only SLM. This method is based on digital computer holography, as discussed in [14]. Here we present the main equations, in order to provide a clear and step-by-step derivation of the MD principle.

A beam at the output of a GRIN MMF may be represented as the superposition of Laguerre-Gaussian (LG) modes [15-17]: it can be formally expressed as

$$
U(\vec{\rho})=\sum_{\text {even,odd }} \sum_{m, p=0}^{\infty} B_{m p} \cdot \psi_{m p}(\vec{\rho}),
$$

where $B_{m p}$ are the complex amplitudes of modes with principal mode number $n=2 p+|m|, m$ is an azimuthal mode parameter, and $p$ is a radial mode parameter, respectively. For the sake of simplicity, we shall omit to explicitly indicate the evenness of modes: in further theoretical considerations and experiments, this is implied by using the '- $m$ ' notation for odd modes. $\psi_{m p}$ are given by

$\psi_{m p}(\vec{\rho})=N_{m p} \rho^{|m|} L_{p}^{|m|}\left(\rho^{2}\right) e^{-\rho^{2} / 2} \begin{cases}\cos (m \phi), & \text { for even modes } \\ \sin (m \phi), & \text { for odd modes }\end{cases}$

where $L_{p}^{|m|}$ are generalized Laguerre polynomials, $N_{m p}=\left(\int_{\mathbf{R}}\left|\psi_{m p}\right|^{2}\right)^{-1 / 2}$-normalized coefficient, $\vec{\rho}=$ $\sqrt{2} \vec{r} / w_{0}$-radius vector normalized to the size of the fundamental mode. Without loss of generality, we may normalize the $B_{m p}$ coefficients as follows: $\sum_{m=-\infty}^{\infty} \sum_{p=0}^{\infty}\left|B_{m p}\right|^{2}=1$. Thus, each $\left|B_{m p}\right|^{2}$ represents the fraction of energy in the corresponding mode, and the total energy of the field $U$ is 1 .

The main objective of MD is that of searching for the complex mode amplitudes. Mathematically, these can be expressed as a simple dot product: $B_{m p}=\left\langle\psi_{m p} \mid U\right\rangle$. In a physical experiment, such an operation can be realised by means of a spatial filter, with a transmission function $T_{m p}(x, y)$ equal to $\psi_{m p}(x, y)$, and a Fourier lens acting as an integrator. At the Fourier plane we measure the intensity distribution (far-field) [18], which can be expressed as:

$$
\begin{aligned}
I_{m p}\left(x^{\prime}, y^{\prime}\right) & =\left|B_{m p}^{\prime}\left(x^{\prime}, y^{\prime}\right)\right|^{2} \\
& =\left|\iint_{-\infty}^{\infty} T_{m p}(x, y) U(\vec{\rho}(x, y)) \mathrm{e}^{\mathrm{i}\left(x x^{\prime}+y y^{\prime}\right)} d x d y\right|^{2}
\end{aligned}
$$

Hence, it follows that $\left.B_{m p}^{\prime}\left(x^{\prime}, y^{\prime}\right)\right|_{(0,0)}=B_{m p}$. That is, the field amplitude at the center of the Fourier plane yields precisely the desired mode amplitude value. Such technique is available when using a spatial filter, that permits to simultaneously encode amplitude and phase information, as described in details in [19]. In that work, MD was performed all-optically, by using a diffractive mode analysing element (MODAN). The MODAN transmission function is proportional to the complex conjugate mode function $\psi_{m p}^{*}$, and it can include multiple patterns with individual frequency shifts, in order to combine in a single picture the information about series of modes. 


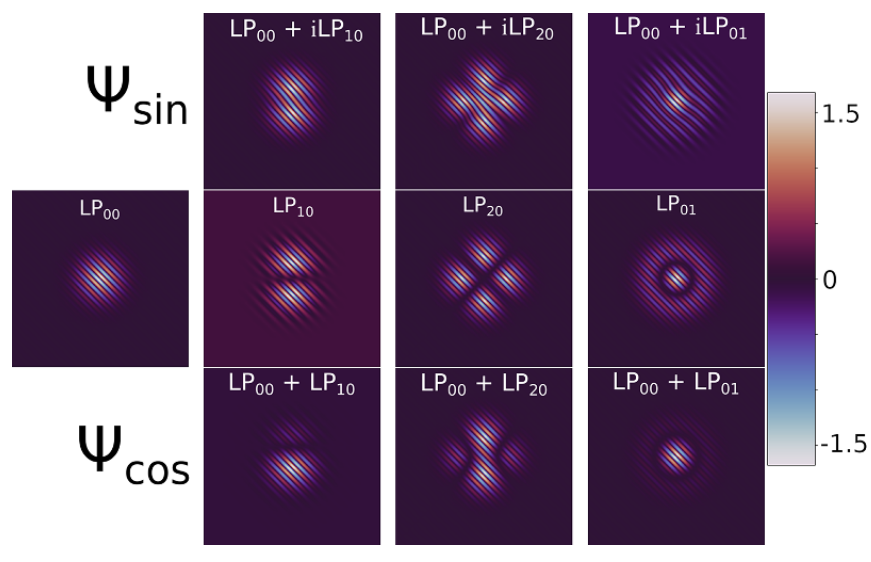

Figure 3. $L G$ modes in the middle plots; superposition of the $L G_{00}$ mode with other modes in the top plots; superposition of the $L G_{00}$ mode and other modes with a $\pi / 2$ phase shift in the bottom plots.

Liquid crystal SLMs are an attractive tool for wavefront control and laser beam shaping. Although they are becoming widely available for research and industry applications, they can only effectively manipulate the phase of a light beam. Specifically, an SLM permits to impose a phase mask $H(x, y)$ on an incident beam:

$$
U_{\text {refl }}=U_{\text {inc }} \mathrm{e}^{\mathrm{i} H(x, y)}
$$

where $U_{\text {inc }}$ and $U_{\text {refl }}$ are the incident and the reflected field, respectively, and $H(x, y)$-is the phase mask encoded at the SLM display. It has been shown in [14] that the issue of amplitude encoding can be solved by taking into account the following property of complex exponents (Jacobi-Anger expansion):

$$
\mathrm{e}^{\mathrm{i} z \sin (\phi)}=\sum_{k=-\infty}^{\infty} J_{k}(z) \mathrm{e}^{\mathrm{i} k \phi} .
$$

As long as the information provided by $\phi$ is contained in highorder Bessel functions, with a maximum that decreases with their order, one may retain only the contribution of the firstorder Bessel function $J_{1}$.

Let us suppose that the desired transmission function reads as:

$$
T_{m p}=\psi_{m p}^{*} / A_{m p}=a(x, y) \mathrm{e}^{\mathrm{i} \Phi(x, y)},
$$

where the coefficient $A_{m p}$ is selected from the maximum of $J_{1}$ as follows: $\max \left\{\left|T_{m p}\right|\right\}=\max \left\{\left|\psi_{m p}\right|\right\} / A_{m p}=\max \left\{J_{1}\right\} \simeq$ 0.58 [20], so that each mode is normalized to the first maximum value of $J_{1}$.

$$
A_{m p}=\max \left\{\left|\psi_{m p}\right|\right\} / 0.58, a \in[0,0.58], \Phi \in[-\pi, \pi] .
$$

In this case, in order to implement the transmission function of equation (8) with the SLM, one needs the phase mask $H(x, y)$ in equation (6) such that

$$
H(x, y)=z \sin (\phi)=f(a(x, y)) \sin \left(\Phi^{\prime}(x, y)\right),
$$

with the condition

$$
f(a)=J_{1}^{-1}(a), \text { and } \Phi^{\prime}=\Phi+2 \pi q \cdot(x-y)
$$

where the spatial frequency $q$ is introduced for imposing a shift to different orders in the spatial frequency domain. To summarize, by substituting equations (10) and (11) into equation (6), and considering equation (7), one obtains

$$
\begin{aligned}
U_{\text {refl }}=U_{\text {inc }} & \sum_{k=-\infty}^{\infty} J_{k}\left[J_{1}^{-1}[a]\right] \mathrm{e}^{\mathrm{i} k \Phi^{\prime}} \Longrightarrow \\
& \Longrightarrow U_{\text {inc }}\left\{a \mathrm{e}^{\mathrm{i} \Phi}\right\} \mathrm{e}^{\mathrm{i} 2 \pi q \cdot(x-y)},
\end{aligned}
$$

where the expression in the curly brackets is equal to $T_{m p}$ in equation (8). By substituting in equation (5) the first term in the previous expression, one obtains the desired dot product at the center of the first diffraction maximum, i.e. $\left(x^{\prime}, y^{\prime}\right)=(-q, q)$.

Examples of phase masks are shown in figure 3 . In the resulting Fourier-transform of equation (6) with the phase mask of equation (10), one obtains the amplitude of each mode at the center of the first-order of diffraction, as follows:

$$
I_{m p} \cdot A_{m p}^{2}=\left|\left\langle\psi_{m p} \mid U\right\rangle\right|^{2}=\left|B_{m p}\right|^{2} .
$$

In order to verify the obtained results, it is necessary to recompose the beam, and compare it with the original one. This task is impossible to achieve, when one only knows the mode amplitudes. Therefore, it is necessary to determine the phase differences between the modes. To search for relative modal phases, we may calculate their inter-modal interference with the fundamental mode, as described in [19]:

$$
\begin{aligned}
I_{m p}^{\sin } \cdot A_{m p}^{\sin ^{2}} & =\left|\left\langle\psi_{00}-i \psi_{m p} \mid U\right\rangle\right|^{2} \\
& =\left|B_{00}\right|^{2}+\left|B_{m p}\right|^{2}+2\left|B_{00}\right|\left|B_{m p}\right| \sin (\Delta \phi),
\end{aligned}
$$

$$
\begin{aligned}
I_{m p}^{\cos } \cdot A_{m p}^{\cos 2} & =\left|\left\langle\psi_{00}+\psi_{m p} \mid U\right\rangle\right|^{2} \\
& =\left|B_{00}\right|^{2}+\left|B_{m p}\right|^{2}+2\left|B_{00}\right|\left|B_{m p}\right| \cos (\Delta \phi)
\end{aligned}
$$

where $\Delta \phi=\phi_{00}-\phi_{m p}, \phi_{m p}=\arg \left(B_{m p}\right)$. Here $A_{m p}^{\text {sin }}$ and $A_{m p}^{\text {cos }}$ are determined similarly to $A_{m p}$ in equation (9), where now the sum of modes is used instead of a single mode. For convenience, we may assume $\phi_{00}=0$. In order to measure $\phi_{m p}$, we also use the above described technique, but instead of $\psi_{m p}^{*}$ in equation (8) we use the contribution from equations (14) and (15). Examples of these masks are shown in figure 3.

It should be noted that the range of phase shifts in equation (10) does not overcome the value of $1.12 \pi$. This is why we can use the second calibration curve (figure 2) for improving the phase resolution. 


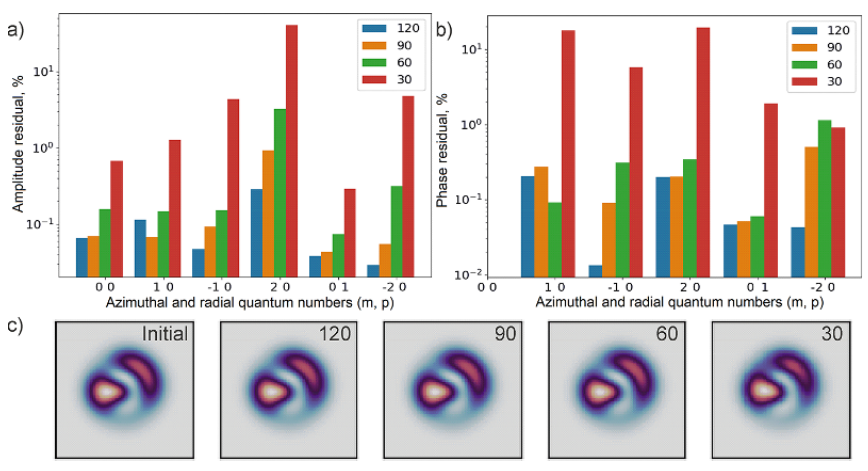

Figure 4. Relative amplitude (a) and phase (b) mismatch in log-scale, calculated in the process of MD simulation; (c) From left to right - original beam; beam reconstructed as a result of decomposition with a fundamental mode size of: 120 pixels; 90 pixels; 60 pixels; 30 pixels.

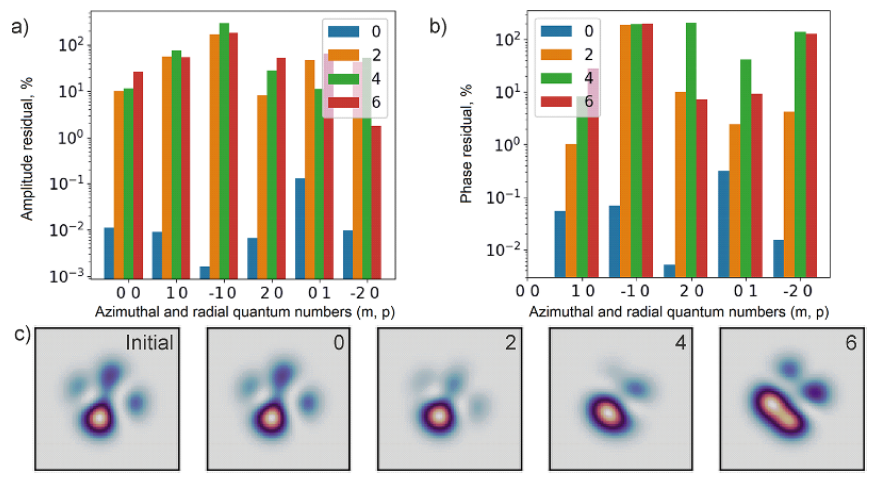

Figure 5. Relative amplitude (a) and phase (b) mismatch in log-scale, calculated in the process of the MD simulation; (c) From left to right - the original beam; a beam reconstructed as a result of MD using 2, 4 and 6 pixel offsets point on camera correspondingly.

\section{Numerical simulations}

In order to verify the derivations, we made numerical simulations of MD by using a phase-only SLM. These also permit us to find out the influence, on the accuracy of our MD method, of factors such as: the spatial resolution in terms of phase mask pixels per fundamental mode size; the value of spatial frequency $q$ in equation (11); the inaccuracy in the first diffraction maximum center as it is measured by the camera. The initial beam consisted of six modes with random amplitudes and intermodal phases. This limited number of modes was sufficient for demonstrating the validity of the method, and for identifying the most critical parameters for the accuracy of the MD. The radius of the fundamental mode was of 120 pixels. The phase was superimposed on the beam as in equation (12), subsequently a Fourier transform was applied to it. Its intensity value was stored at the point $(-q, q)$, corresponding to the spatial frequency shift. We obtained amplitudes and phases of reconstructed beams, and calculated the residual with respect to the original beam, by means of the equation

$$
\left|\left(I_{\text {rec }}-I_{\text {init }}\right) / I_{\text {init }}\right| .
$$

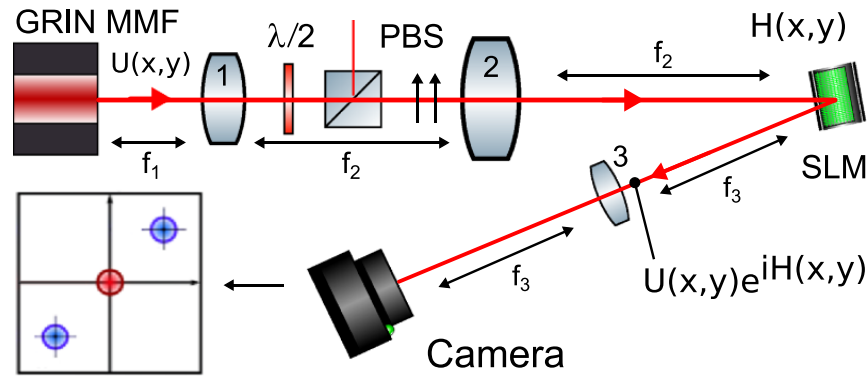

Figure 6. Experimental setup. From GRIN MMF to camera: $50 \mu \mathrm{m}$ core GRIN fiber, aspheric lens, half-wave plate, polarization beam splitter, lens, SLM Pluto-2, Fourier lens, CMOS sensor camera Beamage-4 M.
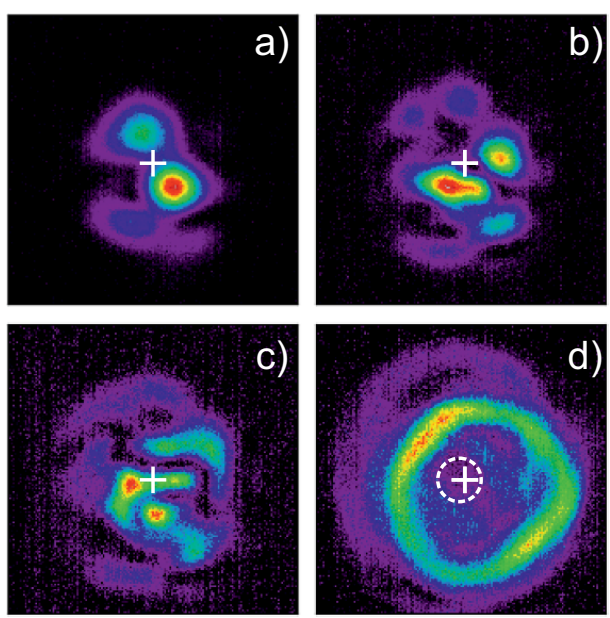

Figure 7. Correlation functions at first-order diffraction maximum: (a) correlation function for $L G_{00}$ mode; (b) for $L G_{01}$ mode; (c) for $L G_{02}$ mode; (d) integrated image (the sum of all) of the first diffraction maximum for higher-order modes.

We started with the investigation of the influence of the phase shift value. For this, the sampling rate of the SLM is an important parameter. If the spatial frequency $q$ is not a multiple of the SLM display sampling rate, the necessary intensity point may be situated in-between the grid points (the so-called picket-fence effect) [21]. If the spatial frequency $q$ is much lower or larger than the SLM sampling rate, the diffraction efficiency will go down. In the MD simulations, we chose as the sampling rate of both the SLM and the camera the value of $f_{s}=1 / 2048$, in order to compare the fidelity of two different MDs, carried out with a spatial frequencies $q$ of either 1/64 or $1 / 57$, respectively. The results of the calculations demonstrate that the relative amplitude mismatch rapidly increases, in cases where the phase shift value is not a multiple of the camera sampling rate.

Next, we compared phase masks with a fundamental mode radius of 120, 90, 60 and 30 pixels, respectively. It was found that the residual of amplitudes and phases increases proportionally with the decrease of the spatial resolution. Whereas, the intensity distributions of reconstructed beams remain qualitatively the same (see figure 4). However, in the case of 30 pixels resolution both residuals grow dramatically, 

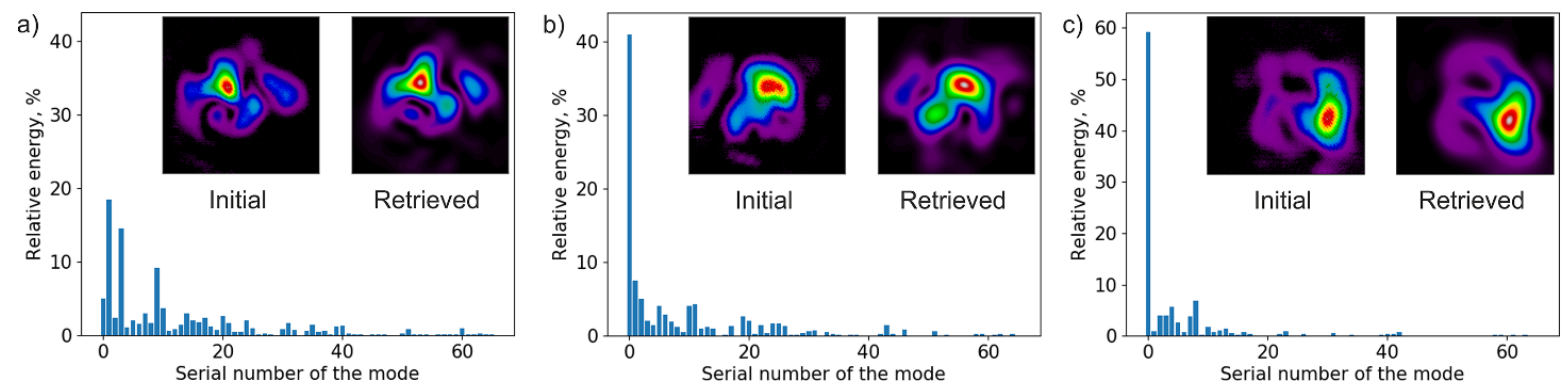

Figure 8. Mode decomposition of three different low-power speckled beams.
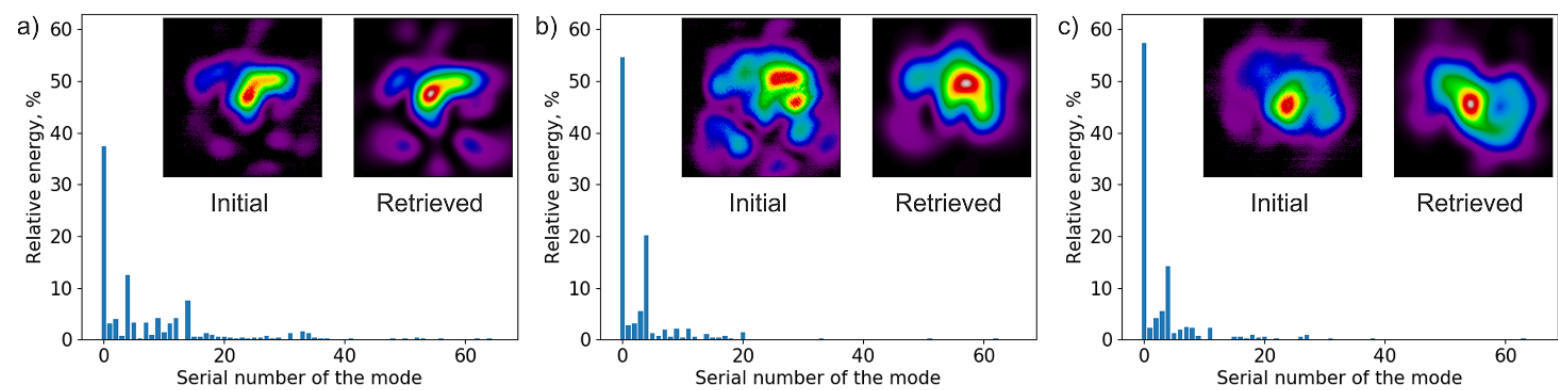

Figure 9. Mode decomposition of the beam with three different input power levels: $0.92 \mathrm{~kW}$ (a), $2.3 \mathrm{~kW}$ (b) and $6 \mathrm{~kW}$ (c).

and this is also accompanied by a slight change in the intensity distribution of the reconstructed beam.

As a final step, we checked the influence of the center offset of the first diffraction order, as it was captured by the camera. We compared the values of the amplitudes and phases, obtained by choosing different points of the first maximum. A sharp increase in the residual was revealed when deviating from the center of the first diffraction maximum, which corresponds to the frequency shift that is specified by the phase mask of the SLM. Such error increase also leads to noticeable differences in the intensity distribution (see figure 5).

\section{Experiment}

A $3 \mathrm{~m}$ long span of standard GRIN MMF with $50 \mu \mathrm{m}$ core diameter was chosen, which permitted a suitable control of the number of excited modes. For this fiber, the fundamental mode radius $w_{0}$ is equal to $6.33 \mu \mathrm{m}$. The measured input beam radius was $15 \mu \mathrm{m}$ (at the $1 / \mathrm{e}^{2}$ of peak intensity level). Lenses 1 and 2 were used to magnify the output beam size at the SLM display, in order to increase the effective resolution. Because SLM only operates in the horizontal polarization, we selected this state of polarization from the input beam. The relative orientation of the half-wave plate and PBS with respect to the fiber was chosen in order to maximize the transmitted energy. The spatial frequency $q$ equation (11) was chosen equal to $1 / 32$, which allows us to save the sinusoidal form of the mask, and effectively separate the high-order Bessel functions in the frequency domain.

In the experiment, we saved and processed images in the Fourier domain. For each mode, we obtained three intensity distributions comprising $I_{m p}, I_{m p}^{\mathrm{sin}}$ and $I_{m p}^{\mathrm{cos}}$, by properly toggling the phase mask on the SLM. Finally, we reconstructed the beam by substituting the calculated $B_{m p}$ in (3).

The beam center position did not change throughout the experiments. We took care to define the point of the first diffraction maximum, where the required intensity value is located. Examples of captured images of the first diffraction maximum are shown on figures 7(a)-(c), where the position of the beam center is not immediately apparent. Therefore, we assumed that high-order modes only contain a relatively small portion of the beam energy, so that our point of interest has minimal intensity for all of them. In figure 7(d) we report integrated images of the first diffraction maximum for several higher-order modes with the largest main quantum number. Since the intensity distribution of the first diffraction maximum is random in our model, and higher-order modes contribute much less than low-order ones, the required point has the lowest level of intensity on these images. By iterating over points from the shaded area, we could obtain the most suitable one.

As a main result of our experiments, we obtained the mode energy distribution of $\sim 80$ modes contributing to the speckled beam. Despite the fact that the used GRIN MMF sustains more than 600 modes at the input wavelength, we found that remaining modes only contain a negligible fraction of power, so that our mode set is sufficient to fully characterize the output beam. To check the validity of our MD, we carried out a successful reconstruction of the beams, based on the retrieved complex amplitudes $B_{m p}$ (see figure 8).

Based on our MD method, we could also reveal the dependence of the output mode energy distribution on the peak power of the input beam. Corresponding histograms, alongside with their near-field intensity distributions, are presented in figure 9. The incident beam peak power was changed from $920 \mathrm{~W}$ up 
to $6 \mathrm{~kW}$, and the coupling ratio was of about 50 percents. As it can be clearly seen by comparing figures 9(a) with (c), the fundamental mode contribution grows from about $38 \%$ to more than $55 \%$ at high powers, which leads to a bell-shaped spatial intensity distribution at the GRIN fiber output [3].

\section{Discussion and conclusions}

To summarize, we demonstrated the successful direct noniterative MD of low-power speckled beams, as well as of highpower beams, consisting of $\sim 80$ modes of a GRIN MMF, for the first time to the best of our knowledge. Numerical simulations of the MD permitted us to clarify the role of the most critical MD parameters, which influence the precision of the reconstruction of the mode amplitudes and of their relative phases. These are: the phase mask size, the spatial frequency shift value, and the center offset of the first diffraction maximum. These findings enabled us to carry out a successful high-fidelity MD, whereby the difference between original and reconstructed beams always remains relatively low. The obtained mode energy distributions permit to conclude that, for low power beams propagating in the linear regime, middleorder modes contain about half of the total beam energy. Whereas at high powers a significant increase of beam energy into the fundamental mode is observed. Small residual inaccuracies in the reconstructed beams indicate that the precision of phase reconstruction could be further improved.

The developed MD technique can be used for the analysis of cleaned beams both via Kerr nonlinearity upon propagation [3] and via the Raman conversion [22] of multimode beams in GRIN fibers, as well as in applications requiring laser beams with a definite composition of transverse modes. In addition, a properly controlled SLM could be used as a mode selective element in high-power fiber amplifiers of ultra-short pulses, based on few- or highly multimode optical fibers.

\section{Acknowledgments}

The study was supported by the Russian Ministry of Science and Education (Grant No. 14.Y26.31.0017). M.G. was also supported by RFBR (Grant No. 20-32-90132), S.W. was also supported by the European Research Council (Grant No. 740355 and Grant No. 874596).

\section{References}

[1] Paschotta R Single-mode fibers (available at: https://www.rp-photonics.com/single_mode_ fibers.html )

[2] Li Z, Zhou J, He B, Lou Q and Xue D 2013 Optik-Int. J. Light. Electron Opt. 124 82-4

[3] Krupa K, Tonello A, Shalaby B M, Fabert M, Barthélémy A, Millot G, Wabnitz S and Couderc V 2017 Nat. Photon. $11237-41$

[4] Lopez-Galmiche G, Eznaveh Z S, Eftekhar M A, Lopez J A, Wright L G, Wise F, Christodoulides D and Correa R A 2016 Opt. Lett. 412553

[5] Krupa K et al 2016 Opt. Lett. 41 5785-8

[6] Li L, Leng J, Zhou P and Chen J 2017 Opt. Express 2519680

[7] Schulze C, Naidoo D, Flamm D, Schmidt O A, Forbes A and Duparré M 2012 Opt. Express 2019714

[8] Flamm D, Naidoo D, Schulze C, Forbes A and Duparré M 2012 Opt. Lett. 372478

[9] Pariente G, Gallet V, Borot A, Gobert O and Quéré F 2016 Nat. Photon. 10 547-53

[10] Nicholson J, Yablon A D, Ramachandran S and Ghalmi S 2008 Opt. Express 16 7233-43

[11] Pourbeyram H, Sidorenko P, Wu F O, Christodoulides D N and Wise F W 2020 Optical thermalization in ultrashort pulse propagation in multimode fiber Conf. Lasers Electro-Optics (Washington D.C.: OSA) p SM1P.6

[12] Forbes A, Dudley A and McLaren M 2016 Adv. Opt. Photon. 8200

[13] Li R and Cao L 2019 Appl. Sci. 92012

[14] Arrizón V, Ruiz U, Carrada R and González L A 2007 J. Opt. Soc. Am. A 243500

[15] Geshiro M, Matsuhara M and Kumagal N 1978 IEEE Trans. Microw. Theory Tech. 26 115-19

[16] Olshansky R 1979 Rev. Mod. Phys. 51341

[17] Trichili A, Park K H, Zghal M, Ooi B S and Alouini M S 2019 IEEE Commun. Surv. Tutorials 21 3175-203

[18] Goodman J W 2005 Introduction to Fourier Optics (Greenwood Village, CO: Roberts and Company Publishers)

[19] Kaiser T, Flamm D, Schröter S and Duparré M 2009 Opt. Express 179347

[20] Flamm D, Schulze C, Naidoo D, Schroter S, Forbes A and Duparre M 2013 J. Light. Technol. 31 1023-32

[21] Oppenheim A V, Buck J R and Schafer R W 2001 Discrete-Time Signal Processing vol 2 (Upper Saddle River, NJ: Prentice Hall)

[22] Kuznetsov A G, Kablukov S I, Wolf A A, Nemov I N, Tyrtyshnyy V A, Myasnikov D V and Babin S A 2019 Laser Phys. Lett. 16105102 